\title{
LIE LOOPS WITH INVARIANT UNIFORMITIES
}

\author{
BY \\ SIGMUND N. HUDSON
}

To Professor Alexander D. Wallace on his sixtieth birthday August 21, 1965.

1. This paper presents generalizations of the theory of Lie groups by weakening the associativity axiom of such algebraic structures. The algebraic-topological structures under investigation are, for the most part, connected, locally euclidean topological loops with a right invariant uniformity and a left invariant uniformity (loops to which we will refer in this introduction as $u$-loops). The first portion of the paper presents partial solutions to a generalized "Hilbert's Fifth Problem," i.e., the problem of introducing analytic structures on $u$-loops so that multiplication, as well as the associated inverse binary compositions, are analytic functions. The last part of this paper assumes the existence of such analytic structures; then the familiar theorems of Lie group theory relating the analytic structures of a Lie group to the algebraic-topological structures of its subgroups and its continuous homomorphisms are shown to hold for Lie loops having various uniformities.

Hilbert's Fifth Problem for locally euclidean groups was first solved in the compact and commutative cases (by von Neumann and Pontrjagin respectively); it was not until techniques reaching deeper into the theory could the general conjecture be verified. In a similar view, we prove the following theorem: $A$ u-loop is a Lie loop if it is either compact or commutative (see Corollary 3.4). Since a topological group has a right invariant uniformity and a left invariant uniformity, this theorem is a generalization of the theorem of von Neumann and Pontrjagin mentioned above. Although in reality we give a somewhat more general result in the body of the paper, the conjecture that a $u$-loop is a Lie loop remains open in general. In the author's opinion, a deeper analysis than given here will be required to solve the problem. Small progress is made in the diassociative case (Theorem 3.6). Included in the results of the latter portion of the paper are the following: If $(L, A),(L, B)$, and $(M, C)$ are Lie loops with respect to the analytic structures $A, B$, and $C$, respectively, if each of $L$ and $M$ has a right invariant uniformity, if $N$ is a closed connected subloop of $M$, and if $f$ is a continuous homomorphism from $L$ to $M$, then $A$ is analytically equivalent to $B, f$ is an analytic mapping with respect to $B$ and $C, N$ is an analytic submanifold of $M$ and also a Lie loop in the natural

Presented to the Society, April 20, 1964; received by the editors March 16, 1964. 
relative analytic structure, and there is a neighborhood $U$ of the identity of $L$ having "unique $n$th roots" and no subloops other than the identity.

The assumption of various invariant uniformities for the loops considered in the paper is important and necessary to obtain the desired conclusions; this remark may be verified by easily constructed examples. The theorems and techniques used are those of Lie groups and Lie transformation group theory. These techniques are applied to certain "groups of translates" which arise from the loops with their various uniformities. Classical theorems in transformation group theory of Montgomery and Bochner, Pontrjagin, and Myers and Steenrod provide the foundations for the strongest results. On one hand, the proofs of our theorems give no new techniques for Lie group theory, and, in fact, the proofs of many of the theorems lean heavily on the validity of the theorem in question for the associative (i.e., the group) case. On the other hand, some of the theorems show that the algebraic assumption of associativity is not as important as it might seem to be.

Most of this paper, along with [6], form the main body of the author's dissertation presented to the Department of Mathematics of the Graduate School of Tulane University in April 1963 under the supervision of Professors Paul S. Mostert and Karl Heinrich Hofmann, to whom the author is most grateful for their suggestions and advice. The author is also grateful for comments from Professor P. T. Church and Dr. Wu Ta-sun.

2. This section is devoted to a brief discussion and review of definitions, terminolgy, and basic theory to be used in the later sections. These concepts may be found in [4], [6], [7], [9]. A topological loop $(L,$.$) is$ a Hausdorff space with a binary operation $\cdot(x, y)=x y$ so that (a) there is an element $1 \in L$ with $1 x=x 1=x$ for all $x \in L$, (b) for each $x$ and $y$ in $L$, there are unique solutions $a$ and $b$ to the equations $a x=y$ and $x b=y$ ( $a$ and $b$ are denoted by $y x^{(-1)}$ and $x^{(-1)} y$, respectively), and (c) the following three functions of $L \times L$ onto $L$ are continuous: $(x, y) \rightarrow x y,(x, y)$ $\rightarrow x y^{(-1)}$, and $(x, y) \rightarrow x^{(-1)} y$. If there is a uniformity $\mathscr{U}$ on $L$ compatible with its topology having a base $\mathscr{B}$ of entourages of $\mathscr{U}$ so that for all $B$ $\in \mathscr{B},(x, y) \in B$ if and only if $(x a, y a) \in B$ for all $a \in L$, then $L$ is said to have a right invariant uniformity $\mathscr{U}$. Similarly one may define a left invariant uniformity and an invariant (both right and left invariant) uniformity. This terminology is consistent with that for topological groups. As in the case of topological groups, if a topological loop $L$ having a right invariant uniformity is metrizable, then $L$ has a metric satisfying $d(x, y)$ $=d(x a, y a)$ for all $x, y$, and $a$ in $L$ (of course $d$ is consistent with the topology for $L$ ). For each $a$ in a loop $L$ the mapping $x \rightarrow x a$, denoted by $R_{a}$ and called the right translate by $a$, is a homeomorphism of $L$ onto itself. 
Definition 2.1. The group $G$ generated by all the right (respectively left, right and left) translates of a loop $L$ in the group of all homeomorphisms of $L$ onto itself is called the group generated by the right (respectively left, all) translates. If $L$ is locally compact, there is a natural topology which we define for these three groups, namely, the $g$-topology (as given by R. Arens). In this case, all of these groups are topological transformation groups of $L$ and, furthermore, act transitively and effectively on $L$. If $L$ has a right invariant (respectively left invariant, invariant) uniformity $\mathscr{U}$, then the group generated by the right (respectively left, all) translates of $L$ is a uniformly equicontinuous collection of homeomorphisms of $L$ with respect to $\mathscr{U}$.

If $G$ is any transformation group acting on a loop, $G_{1}$ will denote the isotropy, or stability, group of $G$ at the identity 1 of $L$, i.e.,

$$
G_{1}=\{g \in G: g(1)=1\} .
$$

Proposition 2.2. If $L$ is a locally compact, connected loop with a right invariant uniformity, then the closure $G^{-}$of the group $G$ generated by the right translates in the group of all homeomorphisms of $L$ onto itself endowed with the g-topology is a locally compact topological transformation group of homeomorphisms acting effectively and transitively on $L$. The mapping $x \rightarrow R_{x}$ is a homeomorphism of $L$ into $G$ and $G^{-}$. $G$ and $G^{-}$are connected. Arcwise connectedness of $L$ implies arcwise connectedness of $G$.

Proof. Verification of the major portion of this proposition may be found in [6, p. 183]. Since the function $C_{R}$ mapping $x$ onto $R_{x}$ is one-toone and continuous, and since the mapping taking $R_{x}$ onto $R_{x}(1)$ is continuous, it follows that $C_{R}$ is a homeomorphism. Thus $C_{R}(L)$ and $G$ are arcwise connected if $L$ is arcwise connected.

Definition 2.3. The group $G^{-}$of Proposition 2.2 is called the group of right translates of $L$ ( contrasted with the group $G$ generated by the right translates of $L$ ).

Remark. There are obvious analogues to Proposition 2.2 in the case that $L$ has, instead of a right invariant uniformity, a left invariant uniformity, or an invariant uniformity. Hence two more definitions may be made, namely, the group of left translates, and the group of all translates of $L$. This remark is applicable to many of the remaining definitions and propositions of the paper. We will not give these analogous statements, since they may be obtained by obvious changes and substitutions of phrases.

Proposition 2.4. If $L$ is a locally compact, connected loop with a right invariant uniformity, if $G$ is the group generated by the right translates of $L$ and $G^{-}$is its group of right translates, then $G_{1}^{-}$is compact. The mapping 
$g G_{1}^{-} \rightarrow R_{g(1)}$ of the coset space $G^{-} / G_{1}^{-}$into $G$ and $G^{-}$is a (global) crosssection. Also $G^{-} / G_{1}^{-}$is homeomorphic with $L$ through the canonical mapping $g G_{1}^{-} \rightarrow g(1)$.

This Proposition 2.4 may be found in [7].

Proposition 2.5. If $L$ is a locally compact, connected, locally connected, finite-dimensional loop with a left invariant uniformity, then the group $G^{-}$ of left translates of $L$ is a connected Lie group acting effectively and transitively on $L$.

Proof. $G^{-}$is a locally compact, transitive, effective transformation group acting on $L$ and $G^{-} / G_{1}^{-}$is homeomorphic to $L$, according to the previous two propositions. Using the paragraph preceding Theorem 9 of $[10$, pp. 68, 69], as well as the proof of Theorem 9 itself and its Corollary 3 , it follows that $G^{-}$is a Lie group. (The important step is to show that $G_{1}^{-}$is a Lie group.)

3. The theorems in this section are motivated by the question of which loops can be given an analytic structure so that they become Lie loops.

Definition 3.1. A Lie loop $(L, A)$ is a connected topological loop which is a manifold and which has an analytic structure $A$ so that the following functions from the product manifold $L \times L$ into $L$ are analytic:

$$
\begin{aligned}
& (x, y) \rightarrow x y, \\
& (x, y) \rightarrow x y^{(-1)}, \\
& (x, y) \rightarrow x^{(-1)} y \quad \text { for } x, y \in L .
\end{aligned}
$$

Analytic structure is to be taken in the equivalent usual senses of Chevalley [1] or Pontrjagin [13] for Lie groups. If $L$ is a group, then the definition agrees with that of Lie groups.

As mentioned in the introduction, a generalization of the established results in topological group theory would be the following:

ConJecture. If $L$ is a connected, locally connected, locally compact, finite-dimensional loop having a right invariant uniformity and also a left invariant uniformity, then $L$ is a Lie loop. In this section we prove that such a loop, if it is additionally either commutative or compact, is a Lie loop. If the loop is additionally diassociative, then we can show that there is a neighborhood of the identity uniquely covered by one-parameter subgroups.

Proposition 3.2. Let $L$ be a topological loop such that (a) the group $G^{-}$ of the translates of $L$ is a Lie group and (b) the mappings $C_{R}: x \rightarrow R_{x}$ and $C_{L}: x \rightarrow L_{x}$ of $L$ into $G^{-}$and the mapping $F:(g, x) \rightarrow g(x)$ of $G^{-} \times L$ into $L$ are analytic mappings with respect to $G^{-}$and the natural quotient analytic structure $A$ on $L \cong G^{-} / G_{1}^{-}$obtained from $G^{-}$. Then $(L, A)$ is a Lie loop. 
Proof. Let id denote the identity function of $L$ onto itself and $I$ the function of $G^{-}$onto itself mapping $g$ onto $g^{-1}$. The following three function diagrams, which are composed entirely of analytic mappings, complete the proof:

$$
\begin{aligned}
& (x, y) \stackrel{C_{L} \times \mathrm{id}}{\longrightarrow}\left(L_{x}, y\right) \stackrel{F}{\longrightarrow} L_{x}(y)=x y, \\
& (x, y) \stackrel{C_{L} \times \mathrm{id}}{\longrightarrow}\left(L_{x}, y\right) \stackrel{I \times \mathrm{id}}{\longrightarrow}\left(L_{x}^{-1}, y\right) \stackrel{F}{\longrightarrow} L_{x}^{-1}(y)=x^{(-1)} y, \\
& (x, y) \stackrel{C_{R} \times \mathrm{id}}{\longrightarrow}\left(R_{x}, y\right) \stackrel{I \times \mathrm{id}}{\longrightarrow}\left(R_{x}^{-1}, y\right) \stackrel{F}{\longrightarrow} R_{x}^{-1}(y)=y x^{(-1)} .
\end{aligned}
$$

THEOREM 3.3. Let $L$ have an invariant uniformity, and let $L$ be connected, locally connected, locally compact, and finite-dimensional. Then $L$ is a Lie loop.

Proof. It is shown that the hypotheses of the previous proposition are satisfied. By Proposition 2.5 (that is, by the invariant-uniformity version of Proposition 2.5), the group of all translates of $L, G^{-}$, is a connected Lie group. Proposition 2.4 yields the fact that $G_{1}^{-}$is compact.

However compactness of $G_{1}^{-}$implies that $L$ may be given a Riemannian metric invariant under the action of $G^{-}[9$, p. 215], that is, the elements of $G^{-}$are isometries with respect to this metric. The analytic structure for this Riemannian metric is the structure $A$ of the previous proposition. It is known that when $L$ has the analytic structure $A$ the mapping $F$ of the previous proposition is analytic [13, p. 292, Princeton edition].

To show that the mappings $C_{R}$ and $C_{L}$ of the previous theorem are analytic, we now use techniques introduced by Myers and Steenrod [12]. There exist elements $p_{i}$ of $L, 1 \leqq i \leqq s$, such that if an isometry $g$ in $G^{-}$ leaves all $p_{i}$ fixed, then $g$ is the identity isometry. The integer $s$ is one more than the dimension of the Riemannian manifold $L$. If $g \in G^{-}$, then there corresponds to $g$ the element $\left(g\left(p_{1}\right), \ldots, g\left(p_{s}\right)\right)$, which belongs to the product analytic manifold $L^{s}$. If we denote this mapping by $T$, then $T$ is an analytic mapping because $F$ is analytic. Furthermore Myers and Steenrod show that $T$ is a homeomorphism [12, p. 413], and the image of $T$ is a regular submanifold of class $C^{1}$ of $L^{s}$ so that the induced multiplication obtained from $G^{-}$is also of class $C^{1}$ in $T\left(G^{-}\right)$[12, p. 415]. It is now shown that the Jacobian matrix of the transformation $T$ at an arbitrary point of $G^{-}$has nonzero determinant. There exists a transformation $T^{\prime}$ of $T\left(G^{-}\right)$ into canonical coordinates $A^{\prime}$ of the first kind so that the transformation $T^{\prime}$ is of class $C^{1}$ [ 13 , Princeton edition, Theorem 47]. But canonical coordinates of the first kind are analytic coordinates. From the uniqueness theorem for analytic structures on Lie groups it follows that $\left(T^{\prime} \circ T\right)^{-1}$ is an analytic transformation. The Jacobian matrix of the transformation 
$\left(T^{\prime} \circ T\right)^{-1} \circ T^{\prime} \circ T$ is the identity matrix and $T$ must have a nonsingular Jacobian matrix, at any point of $G^{-}$. With the aid of $T$ we can now show that $C_{R}$ and $C_{L}$ are analytic. We have first that $x \rightarrow\left(p_{1} x, \cdots, p_{s} x\right)=x^{\sim}$ is analytic, because $L_{p_{i}}$ is analytic for each $i \leqq s$. But $T^{-1}$ restricted to $T\left(G^{-}\right)$is analytic and $T^{-1}\left(x^{\sim}\right)=R_{x} \in G^{-}$. Consequently, $C_{R}$ is analytic. In a dual manner one shows that $C_{L}$ is analytic. Having satisfied the hypotheses of the previous theorem, the proof is completed.

Corollary 3.4. Let $L$ be a connected, locally connected, locally compact, finite-dimensional loop. Let $L$ have a right invariant uniformity and left invariant uniformity. If $L$ is either compact or commutative, then $L$ is a Lie loop.

Proof. With the above hypotheses, $L$ has an invariant uniformity (it is either compact or commutative); so the previous theorem may be applied.

It is interesting that no associativity is required in this theorem and this corollary.

Corollary 3.5 (TO THE PROOF OF THEOREM 3.3). Let $L$ be a locally compact, locally connected, connected, finite-dimensional loop with a right invariant uniformity. If the dimension of $L$ is $q$, then $G^{-}$, the group of right translates of $L$, has dimension less than or equal to $q(q+1)$.

Proof. Using the fact that $G_{1}^{-}$is compact, introducing a Riemannian metric on $L$, and introducing a homeomorphism of $G^{-}$into $L^{q+1}$ the same as the one constructed in Theorem 3.3 , one sees immediately that $\operatorname{dim} G^{-}$ $\leqq \operatorname{dim} L^{q+1}$.

We now give a theorem which indicates that perhaps the invariant uniformity condition of the previous theorem may be weakened in the diassociative case to the condition of having two uniformities, a right invariant one and a left one.

THEOREM 3.6. Let $L$ be a diassociative loop with a right invariant uniformity. Let $L$ be connected, locally connected, locally compact, and finitedimensional. Then there exists a neighborhsod $U$ of the identity such that every element of $U$ lies on a unique local one-parameter subgroup of $L$ and every pair of elements of $U$ is contained in a connected Lie subgroup of $L$.

Proof. The group $G^{-}$of right translates of $\mathrm{L}$ is a connected Lie group. Recall that $C_{R}: L \rightarrow G^{-}$mapping $x$ onto $R_{x}$ is a homeomorphism. Let $V$ be an open neighborhood of the identity in $G^{-}$so that (a) $V^{-}$is compact ("-" means closure), (b) both $V^{-}$and $V^{-2}$ are uniquely ruled by oneparameter subgroups of $G^{-}$, and (c) if $x \in V^{-}$, then the square root of $x$ belongs to $V$. Let $U$ denote the identity component of $\left\{x \in L: R_{x} \in V\right\}$. Then $U$ is a connected neighborhood of 1 in $L$. It will be shown that $U$ has the desired properties. Let $s$ be the mapping of $U^{-}$into $L$ defined by 
$s(x)=x^{2}$. If $x \neq y$ for $x$ and $y$ in $U^{-}$, then $R_{x} \neq R_{y}$; and we have that $x^{2} \neq y^{2}$, when one recalls that (1) $x, y \in U^{-}$imply that $R_{x}, R_{y},\left(R_{x}\right)^{2}$, and $\left(R_{y}\right)^{2}$ all belong to $V^{-2}$, and (2) $\left(R_{x}\right)^{2}=R_{x^{2}}$ and $\left(R_{y}\right)^{2}=R_{y^{2}}$ by disassociativity. Hence $x \neq y$ implies $x^{2} \neq y^{2}$, and $s$ is one-to-one as well as continuous. Thus $s$ is a homeomorphism. We will show that $U$ is contained in $s(U)$. The set $W=s(U) \cap U$ is open in $U$, and $W$ is nonempty. Let $x_{i} \in W$ so that $x_{i} \rightarrow x \in U$. There exist $y_{i} \in U$ so that $y_{i}^{2}=x_{i}$. Since each $y_{i}$ is contained in the compact set $U^{-}$, we may suppose that $y_{i} \rightarrow y \in L$. Thus $y^{2}=x$ and $y \in U^{-}$. However $x \in U$ implies $R_{x} \in V$ (by choice of $U$ ). Thus $R_{y} \in V$ because $\left(R_{y}\right)^{2}=R_{x}$ and condition (c) holds. The following facts imply that $y \in U: y \in U^{-} ; y \in\left\{x \in L: R_{x} \in V\right\} ; L$ is locally connected. But $y \in U$ implies that $x \in W$. Hence $W$ is open and closed in the connected set $U$. Hence $W=U$ and $U \subset s(U)$. Thus $U$ has unique square roots which are also in $U$. For $x \in U$ we may choose elements $x_{i}$ $\in U$ so that $x_{1}=x$ and $x_{i+1}^{2}=x_{i}$. The element $C_{R}\left(x_{i}\right)$ belongs to the oneparameter subgroup containing $C_{R}(x)=R_{x}$. Convergence of $C_{R}\left(x_{i}\right)=R_{x_{i}}$ to the identity of $G^{-}$in the one-parameter subgroup containing $R_{x}$ implies that the closure of the group generated by $\left\{x_{i}: i \geqq 1\right\}$ is a connected group containing $x$ and yields a one-parameter subgroup.

The following special case of a standard and more general argument shows that every pair of elements of $U$ lie in a connected subgroup of $L$. Let $x, y \in L$ and $P_{x}$ and $P_{y}$ be one-parameter subgroups containing $x$ and $y$, respectively. Let $a$ and $b$ be elements of $P_{x}$ and let $c \in P_{y}$. It will be shown that $\left(1^{\prime}\right)(a b) c=a(b c)$. For any integers $p$ and $q$ it is true that $\left(a a^{p / q}\right) c=a\left(a^{p / q} c\right)$ because $a^{1 / q}$ and $c$ lie in a subgroup. Since $\left\{a^{p / q}: p, q\right.$ are integers $\}$ is dense in $P_{x},\left(1^{\prime}\right)$ follows. By symmetry, $\left(1^{\prime}\right)$ holds for any $a, b$, and $c$ in $P_{x} \cup P_{y}$. It follows that $P_{x}$ and $P_{y}$ are together contained in a connected group.

The author had thought that certain loops with a right invariant uniformity might have a neighborhood of the identity covered by local oneparameter subloops which were not groups. But this situation cannot happen, as the following theorem indicates.

THEOREM 3.7. Let $L$ be a locally compact, locally connected, connected, one-dimensional loop with a right invariant uniformity. Then $L$ is a Lie group.

Proof. It suffices to show that $L$ is a group. The group $G^{-}$of right translates of $L$ is a Lie group having dimension smaller than three (by Corollary 3.5). Since such groups have been classified, one of the following situations must occur: (1) $G^{-}$is abelian, or (2) $G^{-}$is nonabelian, is (topologically) euclidean two-dimensional space, and has for compact subgroups only the identity subgroup. In case (1), $G_{1}^{-}$is abelian, implying 
that it contains only the identity element. This implies that $R_{x} R_{y} R_{x y}^{-1}$ is the identity; in other words, $((z x) y)(x y)^{(-1)}=z$ and $(z x) y=z(x y)$. Case (2) cannot occur since $G_{1}^{-}$is compact and $L$ is one-dimensional.

There is a commutative, diassociative loop which is topologically euclidean 3-space but not a Lie loop. This loop was constructed by Hofmann $[4$, p. 152]. It is easy to construct a loop on the euclidean 1 -sphere which is not a Lie loop. Consider first the loop on the complex numbers of norm one defined by

$$
\boldsymbol{e}^{i \alpha} \circ \boldsymbol{e}^{i \beta}=e^{i(\alpha+\beta+f(\alpha)+f(\beta)-f(\alpha+\beta))},
$$

where $f(x)=(\sin (x-\pi / 2)+1) / 4$. This is a Lie loop, without an invariant uniformity. This loop was given incorrectly by the author in $[6, p$. $190]$. By modifying $f$ slightly so that it is no longer real analytic, one obtains a loop on the 1-sphere which is not a Lie loop.

4. It is shown in this section that Lie loops with right invariant uniformities have some of the same analytic and algebraic properties as Lie groups.

Let $(L, A)$ be a Lie loop with a right invariant uniformity. As previously defined, let $G$ and $G^{-}$be the group generated by the right translates and the group of right translates, respectively. By the right-handed version of Proposition $2.5 G^{-}$is a Lie group. $G$ is arcwise connected in $G^{-}$(Proposition 2.2), and $G$ is therefore an analytic subgroup of $G^{-}[14]$. Since $L$ is a manifold, the compact-open and the $g$-topologies are the same for $G^{-}$. The topology of $G$ as an analytic subgroup of $G^{-}$is the associated locally arcwise connected topology [2] (abbreviated as the alac topology). This topology is also described in [15]. With this topology $G$ is a connected Lie group acting effectively and transitively on $L$.

Definition 4.1. Let $(L, A)$ be a Lie loop with a right invariant uniformity. Then $G$, the algebraic group generated by the right translates with the alac topólogy, is called the modified group of right translates of $L$.

The following lemma shows how $G$ acts analytically on $L$. A theorem of Bochner and Montgomery [9] and a classical result found in Pontrjagin [13, German edition] on Lie transformation groups will be used.

LEMMA 4.2. Let $(L, A)$ be a Lie loop with a right invariant uniformity and let $G$ be the modified group of right translates of $L$. Then $G$ acts analytically on $L$, and $G / G_{1}$ (with its natural quotient analytic structure obtained from $G)$ is analytically isomorphic with $(L, A)$.

Proof. We have shown that $G$ is a Lie group of homeomorphisms acting effectively and transitively on $L$. Each element of $G$ is a composition of right translates and their inverses, which are all analytic transformations. The function $F: G \times L \rightarrow L$ defined by $(g, x) \rightarrow g(x)$ is analytic in its second 
variable $x$ for a fixed $g \in G$. The theorem of Bochner and Montgomery $[9$, p. 212] concludes that $F$ is analytic in both of its variables. Thus $G$ acts analytically on $L$. We may now apply the following theorem in Lie transformation group theory [13, German edition, Satz 66]: If $G$ is a Lie group acting effectively, transitively, and analytically on an analytic manifold $(M, A)$, then the canonical homeomorphism from $G / G_{1}$, for $1 \in M$, onto $M$ is an analytic isomorphism, where the analytic structure on $G / G_{1}$ is the natural quotient analytic structure of the coset space. In view of this theorem we have that the analytic structure $A$ is equivalent to the natural analytic structure of the coset space $G / G_{1}$.

We now apply this lemma to closed subloops and homomorphisms of Lie loops with right invariant uniformities.

Theorem 4.3. Let $(L, A)$ be a Lie loop with a right invariant uniformity and let $M$ be a closed subloop of $L$. Then $M$ is a regular analytic submanifold of $L$ and consequently the identity component of $M$ is a Lie subloop.

Proof. Let $H$ be the closure in $G$ (the modified group of right translates of $L$ ) of the group generated by all $R_{x}$ for $x \in M$. Then $H$ is a closed Lie subgroup of $G$ with the relative analytic structure. $H(1)$ is a regular analytic submanifold of $L$ with the quotient analytic structure. But this structure on $L$ is analytically equivalent to $A$. Since $H(1)=M, M$ is a regular analytic submanifold of $(L, A)$.

Theorem 4.4. Let $(L, A)$ and $\left(L^{\prime}, B\right)$ be Lie loops with right invariant uniformities, and let $f$ be a continuous homomorphism from $L$ into $L^{\prime}$. Then $f$ is analytic (relative to $A$ and $B$ ).

Proof. By Theorem $4.3 f(L)^{-}$, the closure of $f(L)$, is a Lie loop in $L^{\prime}$, so that we may assume that $f(L)^{-}=L^{\prime}$ without loss of generality. Let $G$ and $H$ denote the modified group of right translates of $L$ and $L^{\prime}$, respectively. For $x \in L$ define $\phi\left(R_{x}\right)=R_{f(x)}$, and extend the domain of $\phi$ to all of $G$ by the requirement that $\phi$ be a (algebraic) homomorphism from $G$ into $H$.

It will first be shown that $\phi$ is continuous with respect to the compactopen topologies on $G$ and $H$. To accomplish this, it it sufficient to show $\phi$ continuous with respect to the pointwise convergence topologies, since $G$ and $H$ are equicontinuous collections of homeomorphisms as a result of the right invariant uniformities of $L$ and $L^{\prime}[8$, pp. 230 and 232]. Let $\left\{g_{i}\right\}$ be a sequence in $G$ converging to $g \in G$. If $y \in f(L)$, then $y=f(x)$ for some $x \in L$. Then $\phi g_{i}(y)=\phi g_{i}(f(x))=f\left(g_{i}(x)\right)$, the last equality holding by definition of $\phi$. Since $f$ is continuous, $f\left(g_{i}(x)\right)$ converges to $f(g(x))$. But $f(g(x))=\phi g(f(x))=\phi g(y)$. If however $y \in f(L)^{-}$, then there is a sequence $\left\{y_{i}\right\}$ converging to $y$ with each $y_{i} \in f(L)$. We may assume that there is a metric $\delta$ on $L^{\prime}$ which is invariant under the action of $H$. Let 
$\epsilon>0$. We wish to show that there is an integer $N$ such that $n>N$ implies that $\delta\left(\phi g_{n}(y), \phi g(y)\right)<\epsilon$. There is an $N$ such that $n$ and $i$ both greater than $N$ implies that $\delta\left(\phi g_{n}\left(y_{i}\right), \phi g\left(y_{i}\right)\right)<\epsilon / 3$ (because $\left.y_{i} \in f(L)\right)$ and also

$$
\delta\left(\phi g\left(y_{i}\right), \phi g(y)\right)=\delta\left(y, y_{i}\right)=\delta\left(\phi g_{n}(y), \phi g_{n}\left(y_{i}\right)\right)<\epsilon / 3
$$

(because $y_{i}$ converges to $y$ ). We then have

$$
\begin{aligned}
\delta\left(\phi g_{n}(y), \phi g(y)\right)< & \delta\left(\phi g_{n}(y), \phi g_{n}\left(y_{i}\right)\right) \\
& +\delta\left(\phi g_{n}\left(y_{i}\right), \phi g\left(y_{i}\right)\right)+\delta\left(\phi g\left(y_{i}\right), \phi g(y)\right) .
\end{aligned}
$$

The quantity on the right is less than $\epsilon$. Hence $\phi$ is continuous with respect to the pointwise convergence and the compact-open topologies of $G$ and $H$. To show that $\phi$ is continuous with respect to the alac topologies of $G$ and $H$, it is sufficient to show that if $V$ is an open set in the compact-open topology in $H$ and $C$ is an arc-component of $V$, then $\phi^{-1}(C)$ is open in the alac topology for $G$. If $V$ and $C$ are such sets, then $\phi^{-1}(V)$ is open in the compact-open topology for $G$ because $\phi$ is continuous with respect to this topology. If $x \in \phi^{-1}(C)$, then $x \in \phi^{-1}(V)$; and the arc component $D$ of $\phi^{-1}(V)$ containing $x$ is open in $G$ with the alac topology. Furthermore, $\phi(D) \subset C$, because $D$ and hence $\phi(D)$ are connected in the compact-open topologies. Hence $\phi$ is continuous with respect to the alac topologies for $G$ and $H$. From Lie group theory we know that $\phi$ is also analytic.

There exists a local analytic cross section $\pi$ from $U \subset G / G_{1}$ into $G[1]$. Recall that $G / G_{1}$ and $L$ may be identified both topologically and analytically. Next we show that for $x \in U, F^{\prime}(\phi \pi(x), 1)=f(x)$, where $F^{\prime}$ is the analytic mapping of $H \times L^{\prime} \rightarrow L^{\prime}$ defined by $(h, y) \rightarrow h(y)$. For $x \in L$, $\pi(x)$ is a composition of a finite number $n$ of right translates and their inverses. Suppose

$$
\pi(x)=R_{a_{1}}^{t_{1}} R_{a_{2}}^{t_{2}} \cdots R_{a_{n}}^{t_{n}},
$$

for some $a_{j} \in L$, where each $t_{j}= \pm 1, j \leqq n$. Then we have

$$
x=\left(\cdots\left(1 a_{n}^{\left(t_{n}\right)}\right) \ldots\right) a_{1}^{\left(t_{1}\right)} .
$$

Then

$$
F^{\prime}(\phi \pi(x), 1)=\left(\cdots\left(1 f\left(a_{n}\right)^{\left(t_{n}\right)}\right) \cdots\right) f\left(a_{1}\right)^{\left(t_{1}\right)}=f(x),
$$

as claimed. It is now obvious that $f$ is analytic, because $\pi$ is locally analytic, and $F$ and $\phi$ are analytic.

The proof of this theorem comes as a corollary to a later theorem (Theorem 4.6); however, the above techniques may prove useful in the future and are, for this reason, used to prove the theorem.

Similar to Lie group theory, we now obtain the following obvious corollary. 
Corollary 4.5. Let $L$ be a Lie loop with respect to two analytic structures $A$ and $B$. Let $L$ have a right invariant uniformity. Then $A$ is analytically equivalent to $B$.

A crucial step in the proof of Theorem 3.3, i.e., in the introduction of an analytic structure on the loop of Theorem 3.3, was the establishment of the analyticity of the mapping $x \rightarrow R_{x}$ from $L$ into the group of all translates of $L$. The following theorem shows that for Lie loops with a right invariant uniformity this mapping is always analytic.

TheOREM 4.6. Let $(L, A)$ be a Lie loop with a right invariant uniformity. Then the mapping $C_{R}:(L, A) \rightarrow G^{-}$defined by $C_{R}(x)=R_{x}$ is an analytic mapping; and, if $B$ denotes the natural coset-space analytic structure on $L$ obtained from $G^{-} / G_{1}^{-}$, then the identity mapping from $(L, A)$ to $(L, B)$ is an analytic isomorphism.

Proof. The proof uses techniques similar to those of Theorem 3.3. In the following diagram

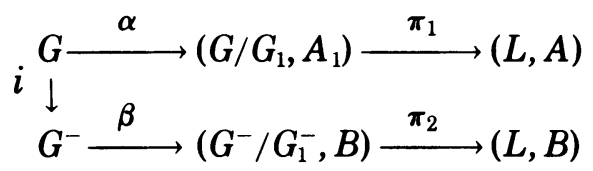

the modified group $G$ of right translates of $L$ and the group $G^{-}$of right translates are Lie groups (see Definition 4.1 and Theorem 2.5), $\alpha$ and $\beta$ are the natural projections and analytic mappings onto the coset spaces $G / G_{1}$ with the natural quotient analytic structure $A_{1}$ and $G^{-} / G_{1}^{-}$with the natural quotient analytic structure $B$, respectively. The canonical mappings $\pi_{1}$ and $\pi_{2}$ are analytic isomorphisms, $\pi_{1}$ by Lemma 4.2. The mappings $\alpha$ and $\beta$, as well as the identity mapping $i: G \rightarrow G^{-}$, are all analytic mappings. We next propose to show that the identity mapping $j:(L, A)$ $\rightarrow(L, B)$ is an analytic function. Because there is a local analytic cross section $p:\left(G / G_{1}, A_{1}\right) \rightarrow G[1$, p. 110$]$, there is a neighborhood $U$ of 1 in $L$ such that $p_{\pi_{1}^{-1}}$ restricted to $U$ is an analytic mapping from the submanifold $U$ into $G$. Furthermore, for each $x \in U$, there is an $h \in G_{1}$ so that $p \pi_{1}^{-1}(x)=R_{x} h$. Hence $\pi_{2} \beta i p \pi_{1}^{-1}(x)=\pi_{2} \beta\left(R_{x} h\right)=x$, that is, $\pi_{2} \beta i p \pi_{1}^{-1}$ agrees with $j$ on $U$. Thus $j$ is locally analytic at $1 \in L$, since the mapping $\pi_{2} \beta i p \pi_{1}^{-1}$ restricted to $U$ is analytic. For any $x \in L$, the set $U_{x}=R_{x}(U)$ is an open set about $x$, and the mapping $R_{x} j R_{x}^{-1}$ from $(L, A)$ onto $(L, B)$ is an analytic mapping locally on $U_{x}$, since $R_{x}^{-1}$ is an analytic isomorphism of $(L, A)$, since $j$ is locally analytic at $1 \in L$, and since $R_{x}$ is an analytic isomorphism of $(L, B)$. Hence $j$ is locally analytic at each element of $(L, A)$, that is, $j$ is an analytic mapping.

In this paragraph we will show that the Jacobian matrix of $j$ at each 
point of $L$ is nonsingular, thereby proving that $j$ is an analytic isomorphism; and in the next paragraph we will show that $C_{R}$ is an analytic mapping. We may assume that there are coordinate systems $Y_{1}$ and $Y_{2}$ both at the identity of $G$, coordinate systems $Z_{1}$ and $Z_{2}$ at the identity of $G^{-}$, and a neighborhood of $1 \in L$, such that the following is true: (1) $r, s$, and $t$ are integers with $r \leqq s \leqq t$, (2) for $x \in V$ with coordinates $\left(x_{1}, \cdots, x_{r}\right)$ in the coordinate system $A, p \pi_{1}^{-1}(x)$ has coordinates $\left(x_{1}, \cdots, x_{r}, 0, \cdots, 0\right)$ in $Y_{1}$, the last $s-r$ coordinates being zero, (3) $p \pi_{1}^{-1}(x)$ has coordinates $\left(y_{1}, \cdots, y_{s}\right)$ in $Y_{2}$ and the function $\tau_{1}$ mapping $\left(x_{1}, \cdots, x_{r}, 0, \cdots, 0\right)$ onto $\left(y_{1}, \cdots, y_{s}\right)$ is an analytic mapping with nonsingular Jacobian matrix $M_{2}$, (4) $i p \pi_{1}^{-1}(x)$ has coordinates $\left(y_{1}, \cdots, y_{s}, 0, \ldots, 0\right)$ in $Z_{1}$, the last $t$-s coordinates being zero, (5) ip $\pi_{1}^{-1}(x)$ has coordinates $\left(z_{1}, \cdots, z_{t}\right)$ in $Z_{2}$ and the function $\tau_{2}$ mapping $\left(y_{1}, \cdots, y_{s}, 0, \cdots, 0\right)$ onto $\left(z_{1}, \cdots, z_{t}\right)$ is an analytic mapping with nonsingular Jacobian matrix $M_{4}$, and finally (6) $\pi_{2} \beta i p \pi_{1}^{-1}(x)$ has coordinates $\left(z_{1}, \cdots, z_{r}\right)$ in the coordinate system $B$. Such coordinate systems exist in Lie groups, their analytic subgroups, and their coset spaces. Let $M_{1}$ denote the $s \times r$ matrix ( $s$ rows and $r$ columns) such that the first $r$ rows form an $r \times r$ identity matrix and all other entries are zero, $M_{3}$ denote the $t \times s$ matrix such that the first $s$ rows form an $s \times s$ identity matrix and all other entries are zero, and $M_{5}$ denote the $r \times s$ matrix such that the first $r$ columns form an $r \times r$ identity matrix and all other entries are zero. Since $j$ is locally $\pi_{2} \beta i p \pi_{1}^{-1}$ at $1 \in L$, the Jacobian matrix of $j$ at 1 is $M_{5} M_{4} M_{3} M_{2} M_{1}$. It is easily verified that this product yields an $r \times r$ nonsingular matrix. Using $R_{x}$ and $R_{x}^{-1}$ as above, it follows that the Jacobian matrix of $j$ at any point of $L$ is nonsingular.

As in the proof of Theorem 3.3, we introduce a Riemannian metric on $(L, B)$ so that the elements of $G^{-}$are isometries of $L\left(G_{1}^{-}\right.$is compact) and choose elements $p_{1}, \cdots, p_{r}$ in some neighborhood of $1 \in L$ so that two elements of $G^{-}$are equal if and only if they agree for all $p_{i}$. We next introduce the following diagram:

$$
(L, A) \stackrel{T_{1}}{\longrightarrow}\left(L^{n}, A^{n}\right) \stackrel{T_{2}}{\longrightarrow}\left(L^{n}, B^{n}\right) \stackrel{T_{3}}{\longleftarrow} G^{-} .
$$

It is understood that if $(M, C)$ is a manifold with analytic structure $C$, then $\left(M^{n}, C^{n}\right)$ means the topological manifold $M^{n}$ with the natural analytic product structure $C^{n}$ obtained from $C$. In this diagram $T_{1}$ is defined by $T_{1}(x)=\left(p_{1} x, \cdots, p_{n} x\right)$; it is an analytic mapping since $L_{p_{i}}$ is an analytic mapping on $(L, A)$. The mapping $T_{2}$ is defined as the identity mapping; it is an analytic isomorphism since $j$ is one. The mapping $T_{3}$ is defined by $T_{3}(g)=\left(g\left(p_{1}\right), \cdots, g\left(p_{n}\right)\right)$. As in the case of Theorem 3.3 , it follows that $T_{3}$ is a homeomorphism and is analytic. It can also be shown that $T_{3}$ has a nonsingular Jacobian matrix at each point of $G^{-}$in a manner the same as that of Theorem 3.3. Hence $T\left(G^{-}\right)$is a regular analytic sub- 
manifold of $\left(L^{n}, B^{n}\right)$. Letting $T_{4}$ denote $T_{3}^{-1}$ restricted to the analytic submanifold $T_{3}\left(G^{-}\right)$, the composite mapping $T_{4} T_{2} T_{1}$ is analytic; furthermore $T_{4} T_{2} T_{1}(x)=R_{x}=C_{R}(x)$. With this conclusion the proof is finished.

The next theorem will provide a neighborhood of the identity of certain loops having unique " $n$th roots." If these loops are monassociative, then one-parameter semigroups may be constructed in the neighborhood. The following lemmas play an important role in the determination of this neighborhood. In the second lemma $s_{n}: G \rightarrow G$ is a mapping defined on a Lie group $G$ by $s_{n}(g)=g^{n}, g \in G(n$ is a natural number).

Lemma 4.7. Let $f: U \rightarrow V$ be a mapping from $U$ into $V$, where both $U$ and $V$ are neighborhoods of the origin $o$ in euclidean $n$-space. Suppose that $f$ is of class 1 in $U$ and $f(o)=0$. If the Jacobian matrix of $f$ at $o$ is $r I_{n}$, where $r$ is an integer at least two and $I_{n}$ is the $(n \times n)$-identity matrix, then for any neighborhood $U^{\prime}$ of o there is a neighborhood $W$ of o, with $W \subset U \cap U^{\prime}$ such that (1) $W \subset f(W)$ and $f(W)$ is a neighborhood of o, (2) for any $x \in f(W)$ there is one and only one $y \in f(W)$ with $f(y)=x$, (3) $x$ and $f(x)$ in $f(W)$ imply that $a\|x\| \leqq\|f(x)\|$, where $a=(3 / 4)(r-1 / 8)$.

Proof. Only an outline of the proof of this lemma is given. There is a neighborhood $W_{1}$ of $o$ such that $f\left(f\left(W_{1}\right)\right) \subset V$. There is also a neighborhood of $o$ such that $f$ is one-to-one on this neighborhood. Finally we will show that $\|f(x)\| /\|x\|$ converges to $r$ as $x$ converges to $o$. By the mean value theorem $f_{i}(x)-f_{i}(0)=(x-o) \cdot \nabla f_{i}\left(t_{i} x\right)$, where $0 \leqq t_{i} \leqq 1, f_{i}(x)$ is defined by $f(x)=\left(f_{1}(x), \cdots, f_{n}(x)\right)$, and $\nabla f_{i}\left(t_{i} x\right)$ is the $i$ th row of the Jacobian matrix of $f$ at $t_{i} x$. Thus

$$
\begin{aligned}
\|f(x)\|^{2} & =\sum_{i=1}^{n} f_{i}(x)^{2}=\sum_{i=1}^{n}\left(x \cdot \nabla f_{i}\left(t_{i} x\right)\right)^{2} \\
& =\sum_{i=1}^{n}\|x\|^{2}\left\|\nabla f_{i}\left(t_{i} x\right)\right\|^{2} \cos ^{2} \phi_{i}=\|x\|^{2} \sum_{i=1}^{n}\left\|\nabla f_{i}\left(t_{i} x\right)\right\|^{2} \cos ^{2} \phi_{i},
\end{aligned}
$$

where $\phi_{i}$ is the angle between $x$ and $\nabla f_{i}\left(t_{i} x\right)$. As $x$ converges to $o, t_{i} x$ converges to $o$, and $\left\|\nabla f_{i}\left(t_{i} x\right)\right\|^{2}$ converges to $r^{2}$. Also $\sum_{i=1}^{n} \cos ^{2} \phi_{i}$ converges to 1 .

Lemma 4.8. Let $G$ be a Lie group with a closed subgroup $H$, and let $\alpha$ be the natural mapping from $G$ onto $H$. Let $p: U \rightarrow G$ be a local differentiable cross-section at $x \in G / H$, where $U$ is a neighborhood of $x$ and $p(x)=e$, the identity of $G$. If $n$ is the dimension of $G / H$ then the Jacobian matrix of $\alpha s_{r} p$ at $x \in G / H$ is $r I_{n}$, where $I_{n}$ is the $(n \times n)$-identity matrix.

Proof. By differentiable we mean the existence of continuous first partial derivatives for $p$ with respect to the natural quotient analytic structure 
on $G / H$ and the analytic structure of $G$. Let $D_{1}$ be a coordinate system in $G$ on a neighborhood $W$ of the identity $e$ of $G$ such that, if $g \in W$ and $g$ has coordinates $\left(x_{1}, \cdots, x_{n}, x_{n+1}, \cdots, x_{k}\right)$, then $\alpha(g) \in U$ and $\alpha(g)$ has coordinates $\left(x_{1}, \cdots, x_{n}\right)$ in the natural coordinate system $D$ in the neighborhood $\alpha(W)$ of $x$ (see [1, p. 110]). Also let $D_{2}$ be a coordinate system on a neighborhood $W^{\prime}$ of $e$ with $W^{\prime} \subset W$, so that the coordinates of elements of $W^{\prime}$ are canonical of the first kind $[1$, p. 118], and so that the identity mapping $i:\left(W^{\prime}, D_{1}\right) \rightarrow\left(W^{\prime}, D_{2}\right)$ is an analytic isomorphism with nonsingular Jacobian matrix $J_{2}(w)$ at each point $w$ of $W^{\prime}$. Finally let $V$ be a neighborhood of $x$ in $G / H$ such that $p(V) \cup s_{r} p(V) \subset W^{\prime}$. The purpose of introducing canonical coordinates $D_{2}$ of the first kind in the neighborhood $W^{\prime}$ is so that the mapping $s_{r}:\left(p(V), D_{2}\right) \rightarrow W^{\prime}$ given in the lemma and restricted to $p(V)$ will have as its Jacobian matrix at each point of $p(V)$ the matrix $r I_{k}$, where $I_{k}$ is the $(k \times k)$-identity matrix, and $k$ is the dimension of $G$. Finally let $J_{1}(v)$ and $J_{4}(p(v))$ denote the Jacobian matrices of $p$ and $\alpha$, respectively, at $v \in V$ and $p(v) \in W^{\prime}$, respectively. Since $\alpha p$ is the identity mapping, $J_{4}(p(v)) J_{1}(v)$ is the $(n \times n)$-identity matrix $I_{n}$. Then the composite mapping $\alpha s_{r} p=\alpha i^{-1} s_{r} i p$ has as Jacobian matrix at $v \in V$ the matrix

$$
J_{4}\left(\alpha s_{r} p(v)\right)\left(J_{2}\left(s_{r} p(v)\right)\right)^{-1} r I_{k} J_{2}(p(v)) J_{1}(v),
$$

which is a nonsingular matrix. We have actually proved more than required in the lemma; the conclusion of the lemma follows by letting $v$ $=x$ in $\left(^{*}\right)$.

TheOREM 4.9. Let $(L, A)$ be a Lie loop with a right invariant uniformity, and let $r$ be an integer at least two. Then for any neighborhood $V$ of 1 there is a neighborhood $U$ of 1 , with $U \subset V$, such that for each $x \in U$ there is one and only one $y$ in $U$ with $R_{y}^{r}(1)=x$. Define for $x \in U, x_{1}$ to be $x$. If $x_{i} \in U$ has been defined, define $x_{i+1}=y \in U$, where $R_{y}^{r}(1)=x_{i}$. Then the sequence $\left\{x_{i}\right\}$ converges to $1 \in U$. The neighborhood $U$ may be so chosen that it has a coordinate system $D$ with respect to $A$ and that $x$ and $R_{x}^{r}(1)$ in $U$ imply that $a\|x\| \leqq\left\|R_{x}^{r}(1)\right\|$, where $a=(3 / 4)(r-1 / 8)$ and the norm is taken with respect to $D$.

Proof. Letting $G^{-}$, the group of right translates of $L$, be the Lie group in Lemma 4.8, and $G_{1}^{-}$be the closed subgroup $H$, letting $x=G_{1}^{-}$and letting $p=C_{R} \pi$, where $\pi$ is the canonical mapping of $G^{-} / G_{1}^{-}$onto $L$ and $C_{R}$ the mapping defined by $C_{R}(x)=R_{x} \in G^{-}$, then it follows that the mapping $f: L \rightarrow L$ which maps $x$ onto $R_{x}^{r}(1)$ has Jacobin matrix $r I_{n}$ at $1 \in L$, for $L$ of dimension $n$. If $U$ and $V$ are coordinate neighborhoods of 1 so that $f(U) \subset V$, then the conclusion follows from Lemma 4.7.

Corollary 4.10. If $(L, A)$ is a Lie loop with a right invariant uniformity, 
then there is a neighborhood $U$ of 1 such that $U$ contains no subloops other than $\{1\}$.

Proof. Choose $r, D, a$, and compact $U$ as in Theorem 4.9. Let $x \neq 1$ be in $U$. Let $z=\max \{\|y\|: y \in U\}$. There is an integer $p$ such that $a^{p}>z$. Let $g$ be defined by $R_{x}^{r}(1)=g(x)$, and let $g^{j}$ be the jth composite of $g$ with itself. Then $g^{j}(x) \notin U$ for some integer $j$ satisfying $2 \leqq j \leqq p$. Consequently, no subloop is contained in $U$ except $\{1\}$.

Recall that a one-parameter semigroup in a loop $L$ is a set $\sigma([0,1])$, where $\sigma:[0,1] \rightarrow L$ is a one-to-one function such that $\sigma(0)=1 \in L$ and that $\sigma(a+b)=\sigma(a) \sigma(b)$ for all $a$ and $b$ in the compact interval of real numbers $[0,1]$ for which $a+b \in[0,1]$ (see [11]). Also recall that a monassociative loop is one in which each of its elements lies in a subsemigroup.

Theorem 4.11. Let $(L, A)$ be a monassociative Lie loop with a right invariant uniformity. Then there is a neighborhood $U$ of 1 in $L$ such that every element of $U$ lies on a unique local one-parameter semigroup in $L$.

Proof. Choose $U$ compact and with the properties of the conclusions of Theorem 4.9 and Corollary 4.10. Let $x \in U$, and define $x_{i} \in U$ as in Theorem 4.9 for $r=2$. Also define $x^{p / 2_{i}^{i}}=x_{i}^{p}$, for natural numbers $p$ and $i$ with $p / 2^{i} \leqq 1$. The product $x_{i}^{p}$ is well-defined by monassociativity. Let $J=\left\{x^{p / 2^{i}}: p / 2^{i} \leqq 1\right\}$. Standard one-parameter techniques (see, e.g., [3] and [5]) show that $J^{-}$is a local one-parameter semigroup containing 1 and $x$. One uses the following line of argument: (1) $J^{-}$is a local commutative, locally compact semigroup containing 1 and $x ;(2)$ if $\left\{t_{i}\right\}$ is a sequence of dyadic rational numbers with $0 \leqq t_{i} \leqq 1$ converging to zero such that $\lim _{i} x^{t_{i}}=z$, then $z=1$; (3) if $s$ is a real number, and $\left\{s_{i}\right\}$ and $\left\{t_{i}\right\}$ are two sequences of dyadic rational numbers converging to $s$ with $0 \leqq s_{i} \leqq 1$ and $0 \leqq t_{i} \leqq 1$, then $\lim _{i} x^{t_{i}}$ and $\lim _{i} x^{s_{i}}$ exist and are equal; (4) with $s$ and $s_{i}$ as in (3), define $x^{s}=\lim _{i} x^{s_{i}}$, hence, the function $t:[0,1] \rightarrow L$ given by $t(s)=x^{s}$ is well-defined; (5) $t$ is continuous, a homomorphism, one-to-one, and $t(0)=1, t(1)=x$.

We remark that, if $L$ is not only monassociative but powerassociative, then the one-parameter semigroups can be extended to one-parameter subgroups. Recall that a compact, monassociative loop is powerassociative.

\section{REFERENCES}

1. C. Chevalley, Theory of Lie groups, Princeton Univ. Press, Princeton, N. J., 1946.

2. A. M. Gleason and R. Palais, On a class of transformation groups, Amer. J. Math. 79 (1957), 631-648.

3. A. M. Gleason, Groups without small subgroups, Ann. of Math. (2) 56 (1952), 193-212.

4. K. H. Hofmann, Topologische Loops; Topologische Loops mit schwachen Assoziativitätsforderungen; Topologische Doppelloops, Math. Z. 70 (1958), 13-37; ibid. 70 (1958), 125-155; ibid. 70 (1958), 213-230. 
5. __ Topologische Halbgruppen mit dichter submonogener Unterhalbgruppen, Math. Z. 74 (1960), 232-276.

6. S. N. Hudson, Topological loops with invariant uniformities, Trans. Amer. Math. Soc. 109 (1963), 181-190.

7. _ Transformation group theory in the theory of topological loops, Proc. Amer. Math. Soc. 15 (1964), 872-877.

8. J. L. Kelley, General topology, Van Nostrand, Princeton, N. J., 1955.

9. D. Montgomery and L. Zippin, Topological transformation groups, Interscience, New York, 1955.

10. P. S. Mostert, Sections in principal fibre spaces, Duke Math. J. 23 (1956), 57-72.

11. P. S. Mostert and A. L. Shields, One-parameter semigroups in a semigroup, Trans. Amer. Math. Soc. 96 (1960), 510-517.

12. S. B. Myers and N. Steenrod, The group of isometries of a Riemannian manifold, Ann. of Math. (2) 40 (1939), 400-416.

13. L. Pontrjagin, Topological groups, Princeton Univ. Press, Princeton, N. J., 1939 (Topologische Gruppen, Teubner, Leipzig, 1958).

14. H. Yamabe, On an arcwise connected subgroup of a Lie group, Osaka Math. J. 2 (1950), 13-14.

15. G. S. Young, The introduction of local connectivity by change of topology, Amer. J. Math. 68 (1946), 479-494.

\section{SYRACUSE UNIVERSITY,}

SYracuSE, New YorK 